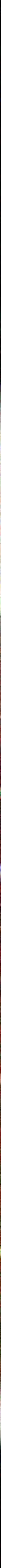




\section{Cryoelectron Tomography}

Stephen W. Carmichael, ${ }^{1}$ Mayo Clinic carmichael.stephen@mayo.edu

We have all seen beautiful three-dimensional (3D) renderings of molecular models, complete with helices and pleated sheets. And for more than half a century we have also seen electron micrographs of cellular structures, although usually in two dimensions. There has been a gap between these two sets of images. By developing a technique that promises $3 \mathrm{D}$ images at resolutions approaching the molecular level, Ohad Medalia, Igor Weber, Achilleas Frangakis, Daniela Nicastro, Günther Gerisch, and Wolfgang Baumeister have bridged that gap. ${ }^{2}$ The technique is called cryoelectron tomography (cryo-ET). Small specimens, typically a cell, are flash-frozen (vitrified) to literally freeze their structure in position. Using a special apparatus, the specimens are robotically rotated about an axis perpendicular to an electron beam while a series of 2-dimensional digital images are obtained. The efficiency of the data acquisition is key, because it minimizes the exposure of the specimen to the electrons.

Whereas the basics of this technique have been appreciated for decades, there were two competing limitations. Resolution and quality of a tomogram are directly dependent on spacing of the acquired images and on the angular range covered; that is, more images acquired closer together over a greater angle is better, but requires more exposure. On the other hand, beams of electrons damage biologic specimens. The key to solving this discrepancy, as mentioned above, is the efficient automated data acquisition. Such methods were developed over the past decade. ${ }^{3}$ Medalia et al. have demonstrated, for the first time, that enough data can be obtained, without damaging the specimen, to reconstruct wonderfully detailed 3D images of an intact cell.

As their biologic specimen, Medalia et al. chose the soil-dwelling amoeba Dictyostelium discoideum. This one-celled animal is a well-utilized model for cell motility, cytokinesis, and other cellular functions. They could visualize actin filaments, membranes, and particles (mostly ribosomes) in situ with amazing spatial fidelity. Two items of particular note were the reliable identification of a macromolecular complex called the $26 \mathrm{~S}$ proteasome, and the angles of attachments of the actin filaments to each other and to membranes. This same group is working on automating the search for particles that have a characteristic shape, such as the $26 \mathrm{~S}$ proteasome, using a template-matching algorithm. The observation that the length of the actin filaments was about twice the commonly accepted length suggests that cryo-ET provides a more faithful image of filamentous structures. Furthermore, the angles of attachment of actin filaments fell into distinct groups, suggesting that certain filament-binding proteins are responsible for certain attachment angles. It was proposed that mutant cells, known to be missing a filament-binding protein, could be examined by cryo-ET to see if a specific angle of attachment was absent, demonstrating that that protein was responsible for that angle.

This new way of looking at cells is certain to have an impact. Cellular substructure can be faithfully visualized in situ with resolution currently in the 5 to $6 \mathrm{~nm}$ range, but resolution of about $2 \mathrm{~nm}$ is possible. This successfully bridges the gap between molecular modeling and conventional electron microscopy, as well as sophisticated optical techniques.

Finally, cryo-ET was selected by the Editors of Science magazine to be on the "top ten" list of breakthroughs during 2002, including all aspects of science. Cryo-ET is sure to open new windows into subcellular structure!

\section{References}

1 The author gratefully acknowledges Dr. Wolfgang Baumeister for reviewing this article.

2 Medalia, O., I. Weber, A.S. Frangakis, D. Nicastro, G. Gerisch, and W. Baumeister, Macromolecular architecture in eukaryotic cells visualized by cryoelectron tomography, Science 298:1209-1213, 2002.

3 Koster A.J., R. Grimm, D. Typke, R. Hegerl, A. Stoschek, J. Walz and W. Baumeister, Perspectives of molecular and cellular electron tomography, J. Struct. Bio1.120:276-308, 1997

\section{InDax or ARTIOLS}

Cryoelectron Tomography Stephen W. Carmichael, Mayo Clinic

Optimizing the Sampling Design of Morphometric Experiments .......5 John M. Basgen, University of Minnesota

FTIR Hyperspectral Images of Microscopic Droplets of Splattered

Blood... Scott W.Huffman*, Kara B. Lukasiewicz ${ }^{* *}$ and Chris W. Brown ${ }^{* \star *}$ ${ }^{*} \mathrm{NIH}$, ${ }^{* *}$ Mayo Clinic, ${ }^{* * *}$ University of Rhode /sland

Setting White, Black and Gamma on Continuous Tone Grayscale and

Color Images using Photoshop .........................................................16 Jerry Sedgewick, University of Minnesota

How to Recognize and Avoid AFM Image Artifacts Paul West and Natalia Starostina, Pacific Nanotechnology, Inc.

Quality in Electron Microscopy. Tony Bruton*, Steve Chapman ${ }^{\star \star}$ and Paul Harding ${ }^{\star * *}$ ${ }^{*}$ E.M. Unit, University of Natal, **Protrain Courses, ${ }^{* \star \star} /$ Integrated Systems Management, Nissan Motor Co.

Use of a New Imaging Technique to Document Deformations Recorded in the Environmental Scanning Electron Microscope ........................36 Edward F. O'Neil, ${ }^{*}$ Hamlin M. Jennings, ${ }^{* \star}$ and Jeffrey J. Thomas ${ }^{* *}$ *US Army Corps of Engineers, "*Northwestern University

On The Limits Of Limits. 39 Tobias I. Baskin, Division of Biological Sciences, University of Missouri Examination of Hydrated Bacteria in An Environmental Scanning

Electron Microscope (ESEM). .....42 Ann Fook Yang and Miloslav Kaláb, Agriculture and Agri-Food Canada
Dye is Money .

Christian Lohr, University of Kaiserslautern, Germany

Water Recirculator (Chiller) Maintenance .........................................43 Owen Mills, Michigan Technological Institute

EDS Spectral Artifacts / Sum Peaks: A Reminder. .43 Steven S. Hurban, Endicott Interconnect Technologies, Inc.

NetNotes.

Industry News

Index of Advertisers

\section{ABOUT THE OOVER}

This image was made using polarized transmitted light in which a 1/4-wave retarder plate (632 Angstroms) was placed in the beam to adjust the color. The slide was prepared by mixing roughly equal parts of Resorcinal and Carbon Tetrabromide powders and placing a small amount of the mix under a cover glass. This was heated slowly to a melt, then allowed to crystalize under natural cooling. The photograph was made by Prof. John Hart, Program in Atmospheric and Oceanic Sciences, University of Colorado, as part of a lecture demonstration on the beauties of chaotic phase changes. Further photographic work by Dr. Hart can be found at http://www.crystalcanyons.net. This image placed nineteenth in the 2001 Nikon Small World Photomicrography contest. 